Onkologe 2019· 25 (Suppl 1):S109-S115 https://doi.org/10.1007/s00761-019-00652-1 Online publiziert: 12. August 2019

(c) Der/die Autor(en) 2019

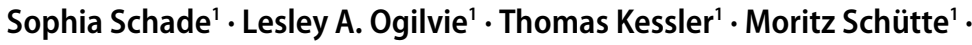
Christoph Wierling ${ }^{1}$ Bodo M. Lange ${ }^{1} \cdot$ Hans Lehrach $^{1,2} \cdot$ Marie-Laure Yaspo $^{1,2}$

${ }^{1}$ Alacris Theranostics $\mathrm{GmbH}$, Berlin, Deutschland

${ }^{2}$ Max Planck Institute for Molecular Genetics, Berlin, Deutschland

\title{
Ein daten- und modellgesteuerter Ansatz zur Behandlung maligner Tumoren
}

\begin{abstract}
Wir sind alle unterschiedlich, mit unterschiedlichen Genomen, unterschiedlichen Anamnesen, unterschiedlichen Lebensstilen und Gewohnheiten und - unter molekularen Aspekten - unterschiedlichen Erkrankungen. Es ist daher nicht erstaunlich, dass wir oft unterschiedlich auf Medikamente reagieren. Um dieser Herausforderung in der Onkologie zu begegnen, brauchen wir viel tiefer reichende Daten zu einzelnen Tumoren und einzelnen Patienten (etwa eine umfassende molekulare Tumoranalyse, "comprehensive molecular tumor analysis" [CMTA]) sowie eine deutlich bessere personalisierte Vorhersage der Auswirkungen möglicher Therapien, zunächst durch Präzisionsmedizin, dann aber zunehmend mithilfe digitaler Modelle einzelner Tumoren und Patienten, mithin über unsere "digitalen Zwillinge".
\end{abstract}

\section{Krebsmanagement ist komplex}

Auf der ganzen Welt sind Krebserkrankungen nach wie vor von erheblicher sozioökonomischer Bedeutung. Jedes Jahr gibt es in Europa etwa 3,7 Mio neue Fälle und 1,9 Mio. krebsbedingte Todesfälle [1]. Jährlich werden allein in Deutschland mehr als 500.000 Patienten neu diagnostiziert und 200.000 versterben an ihrer Krebserkrankung. Weniger als $50 \%$

Die englischsprachige Version des Beitrags ist unter https://doi.org/10.1007/s00761-0190624-z publiziert. der deutschen Krebspatienten überleben länger als 10 Jahre. Die wirtschaftliche Belastung ist enorm: In Europa verursacht Krebs jedes Jahr Kosten von mehr als 126 Mrd. $€$ [2]. Ein erheblicher Teil davon wird für Medikamente ausgegeben, die oft nur einem kleinen Anteil der Patienten helfen.

Ein Grund für diese Zahlen ist zum Teil die Tatsache, dass Krebserkrankungen vielfach erst in späten Stadien diagnostiziert werden, erst, wenn schon weitere Organe betroffen sind und der Tumor eine höhere molekulare Heterogenität erreicht hat, was das klinische Management schwieriger und komplexer macht. Ein weiteres Problem im $\mathrm{Zu}$ sammenhang mit krebsbedingter Mortalität ist die begrenzte Anzahl verfügbarer, zugelassener Medikamente und der beschränkte Zugang zu klinischen Studien. Hinzu kommt, dass jeder Patient und jeder Tumor anders ist - jeder reagiert unterschiedlich auf eine Therapie. Wie effektiv eine Behandlung ist, hängt von der Kombination einzelner patienteneigener und tumorspezifischer Faktoren ab (z. B. Alter, Allgemeinzustand, genetisches Profil, „,molekulare Landschaft" und biologische Eigenschaften des Tumors) sowie von den Wechselwirkungen im Patienten (z. B. Pharmakogenetik, intestinale Mikrobiota, Status des Immunsystems). Detaillierte und genaue Kenntnisse über den einzelnen Tumor und den Patienten sind für die Implementierung effizienter präzisionsmedizinischer Ansätze unerlässlich.

Während die Einzigartigkeit jedes Individuums heute in einigen Berei- chen der Medizin berücksichtigt werden kann, etwa in der Chirurgie, wird sie im Bereich der Pharmakotherapie noch nicht hinreichend adressiert, auch wenn bei der Patientenstratifizierung zweifellos schon wesentliche Fortschritte erzielt worden sind. Insbesondere die Entwicklungen von Profiling-Methoden der molekularen Charakterisierung, vor allem in der Genomik, aber auch der Transkriptomik und der Proteomik, ermöglichen nicht nur Einblicke in die Pathomechanismen des Krebses und die Identifizierung von Patientengruppen, die auf eine bestimmte Therapie ansprechen. Sie zeigen darüber hinaus das Ausmaß der Heterogenität maligner Zellen innerhalb des einzelnen Krebses sowie über Menschen und Populationen hinweg. Präzisionsmedizinische Ansätze, die auf der Charakterisierung des Krebses eines Individuums auf molekularer Ebene basieren, sind für einige Tumorarten schon auf dem Weg in den Mainstream der klinischen Praxis und geben so Krebspatienten neue Perspektiven.

\section{State-of-the-Art: Präzisions- onkologie}

Die Stratifizierung von Patienten mit Biomarkern als Begleitdiagnostik für zielgerichtete Therapien hat die Erfolgsraten onkologischer Therapien erhöht und stellt einen echten Paradigmenwechsel in der klinischen Praxis dar. Um somatische Veränderungen in einer Tumorbiopsie zu erkennen, werden in der Klinik am häufigsten Einzel- 
und Multi-Gen-Panel-Sequenzierungen eingesetzt. So wird beispielsweise der humane epidermale Wachstumsfaktorrezeptor 2 (HER2), ein Protoonkogen, das für den Tyrosinkinaserezeptor HER2 (alias ERBB2) kodiert, als Biomarker bei der Auswahl von Behandlungsoptionen herangezogen. Die Verwendung von HER2-relevanten Wirkstoffen (z.B. die monoklonalen Antikörper Trastuzumab und Pertuzumab) hat das Outcome für Brustkrebspatientinnen in allen Stadien der Krankheit erheblich verbessert [3, 4]. Diese Methoden haben das therapeutische Repertoire erweitert, indem sie die Stratifizierung von Patienten in Untergruppen potenzieller Responder ermöglichen. Sie testen jedoch nur eine begrenzte Anzahl von Genen, nämlich die, welche auf dem Panel vorhanden sind, und bieten nach wie vor nur eine Behandlungsmöglichkeit für diejenigen Patienten, welche diese ausgewählten Marker aufweisen. Das ist in der Regel nur ein kleiner Teil: $25 \%$ der Brustkrebspatientinnen, beispielsweise, überexprimieren HER2, lediglich bei $15 \%$ der Lungenkrebspatienten bestehen Veränderungen im epidermalen Wachstumsfaktorrezeptor (EGFR) oder im Rezeptor-Tyrosinkinase-Protoonkogen 1 (ROS1). Insofern ist die Gesamtwirkung einzelner Biomarkerstrategien begrenzt [5]. Darüber hinaus können zusätzliche individuelle, für jeden Tumor charakteristische genetische Veränderungen mit einer Resistenz gegen die biomarkerselektierte Therapie verbunden sein. So sprechen beispielsweise nur $20-50 \%$ der Patienten mit ERBB2Amplifikation tatsächlich auf Trastuzumab an. Auch die Tumorheterogenität stellt eine weitere Herausforderung dar: Das Ausmaß des Ansprechens auf ein Medikament wird von Zellpopulationen bestimmt, die unterschiedliche biologische Merkmale aufweisen [6]. Groß angelegte Sequenzierungsinitiativen, wie das International Cancer Genome Consortium (ICGC) und der Cancer Genome Atlas (TCGA), charakterisieren die molekulare Komplexität vieler Krebsarten sehr detailliert. Dabei zeigt sich, dass die Identifizierung einzelner essenzieller Treibermutationen („single key driver mutations") bei bestimmten Krebstypen vielleicht gar nicht möglich ist. Diese Bemühungen haben eine Verlagerung des Fokus' ausgelöst: (weg) von einzelnen Treibergenen hin zu einer umfangreicheren und heterogeneren "Mutationslandschaft" (z. B. $[7,8])$. Durch die Beschränkung der molekularen Analyse auf nur wenige Bereiche des Tumorgenoms besteht bei den meisten Präzisionsonkologieprogrammen (die typischerweise Krebsgen-Panels scannen) das Risiko, dass sehr wichtige, möglicherweise für die Therapieentscheidung relevante Informationen nicht untersucht werden. Zusätzliche Omics-Methoden, wie die Transkriptomsequenzierung (RNAseq), bieten wertvolle Erkenntnisse zur Orientierung für Behandlungsempfehlungen, etwa die MammaPrint-Signatur zur Vorhersage des Behandlungserfolgs bei Brustkrebs [9]. RNAseq ermöglicht den Zugang zu einem tiefergehenden Wissen über die Tumorbiologie (u. a. onkogene Veränderungen in der Genexpression, epigenetische Effekte, Spleißvarianten, unerwartete Genfusionen etc.; $[10,11]$ ) und die Tumormikroumgebung, ein stetig wichtiger werdendes Gebiet für klinische Interventionen (z. B. mit Medikamenten, die auf Hypoxie abzielen). Der Durchbruch der Immuncheckpointinhibitoren (ICIs) hat die "Landschaft" der Präzisionsmedizin verändert [12] und zeigt, dass der Fokus der zielgerichteten Therapien weit über mutierte Formen von Krebsgenen hinausgeht - in die Umgebung der Immunabwehr hinein. Ein Prädiktor für das Ansprechen auf ICIs ist die Tumormutationslast (,tumor mutation burden“, TMB). Die TMB lässt sich mit kleinen Genpanels nicht einschätzen. Mehr als 50\% der kutanen Melanome, die typischerweise mit einer großen Anzahl von somatischen Mutationen einschließlich BRAF V600E („proto-oncogene B-Raf“, ,, $\mathrm{V}$-Raf murine sarcoma viral oncogene homolog B1") assoziiert sind, zeigen eine dauerhafte Reaktion auf ICIs mit einer relativ hohen Ansprechrate auf die Anti-PD1 („programmed cell death protein $1^{\prime \prime)}$ Therapie [13]. In diesem Zusammenhang wird die Identifizierung von Biomarkern, die eine Vorhersage der Reaktion auf die Blockade des Immun-Checkpoints erlauben, bei der Auswahl personalisierter
Behandlungsoptionen immer wichtiger. Bei fortgeschrittenen kolorektalen Karzinomen, beispielsweise, ist das Ansprechen auf die Anti-PD1-Therapie generell gering, doch eine Subpopulation von Patienten mit hoher TMB und genomischer Instabilität spricht auf die Therapie an. Daher wird eine eingehende molekulare Analyse des Tumors und seiner Mikroumgebung die Auswahl von immunologischen und zielgerichteten Therapieoptionen erleichtern und neue Möglichkeiten zur Identifizierung relevanter Biomarker für die Vorhersage von Therapieerfolgen und Nebenwirkungen aufzeigen. Aktuelle Daten belegen darüber hinaus, dass Kombinationstherapien im Allgemeinen effektiver sind [14]. Dieser Trend hin zu einer immer detaillierteren Charakterisierung des Tumors (und des Patienten) - von einzelnen Genmutationen und Genpanels hin zu vollständigen Exomsequenzierungsanalysen (,whole exome sequencing", WES) - hat in manchen klinischen Einrichtungen bereits stattgefunden. Doch die Integration von RNAseq-Daten ist zurzeit nur selten implementiert.

In Zusammenarbeit mit dem Charité Comprehensive Center, Berlin, haben wir das vom Bundesministerium für Bildung und Forschung (BMBF) geförderte Pilotprojekt Treat20plus aufgelegt, das auf die tiefgehende molekulare Charakterisierung und Modellierung der Tumoren von Patienten mit metastasierendem Melanom fokussiert ist. Wir haben dafür die CMTA entwickelt, welche die partielle Sequenzanalyse des Genoms, die tiefe Exomsequenzierung sowie eine tiefe Bulk-Tumor-Transkriptomanalyse des Tumors integriert (• Abb. 1) und kombinierte Daten in einem umfassenden und dennoch prägnanten Bericht für das klinische Tumorboard aufbereitet. Diese Daten werden auch zusammen mit der routinemäßigen Tumorpathologie und innovativen Technologien interpretiert, wie etwa mit der bildgebenden CyTOF („cytometry by time of flight")-basierten Massenzytometrie. Wir verwenden das Hyperion-basierte hochmultiplexierte (40 Antigene parallel) System, das es ermöglicht, mehr Informationen über die Heterogenität des Tumors, einer wesentlichen Determinante für die Re- 
zidivwahrscheinlichkeit, und über die Tumormikroumgebung, insbesondere über Vorkommen, Lage und Aktivität der verschiedenen Arten von Immunzellen, zu erhalten. Wir planen zusätzlich eine routinemäßige Bestimmung des Immunstatus' durch emulsionsbasierte Techniken [15] bzw. Einzelzelltranskriptomsequenzierung zirkulierender Immunzellen. Ziel dabei ist es, die Faktoren besser $\mathrm{zu}$ verstehen, welche die wahrscheinliche Reaktion auf verschiedene Immuntherapien beim einzelnen Patienten sowie auch deren Nebenwirkungen bestimmen.

\section{Vorhersage des Ansprechens auf Medikamente in der Präzisionsmedizin}

Basierend auf einer tiefer gehenden molekularen Analyse des Tumors können manche Therapien in Betracht gezogen und andere verworfen werden. Therapievorschläge beruhen dann auf kausalen Argumenten (z. B. Verfügbarkeit eines Medikaments gegen ein Fusionsprotein, das den Tumor antreibt) oder auf Korrelationen spezifischer Biomarker bzw. Signaturen mit (Nicht-) Ansprechen auf unterschiedliche Medikamente. Während einige der „verwertbaren“ Varianten (Mutationen, spezifische Fusionsgene oder Transkripte) bei bestimmten Tumoren häufig vorkommen (das impliziert eine gute Grundlage für die Panelsequenzierung), treten sie bei anderen Tumoren oft mit geringerer Frequenz auf. Solche therapierelevanten Veränderungen werden oft nur durch Analysen erkannt, welche in die Tiefe und in die Breite gehen, also durch Ansätze, wie sie die CMTA und ähnlich umfassende Methoden ermöglichen.

Angesichts der enormen Komplexität des Körpers und seiner Erkrankungen sowie angesichts der vielen Faktoren, welche das Ansprechverhalten auf Medikamente beeinflussen können, ist es wahrscheinlich, dass für eine wirklich personalisierte Therapiewahl mechanistische oder hybride Computermodelle erforderlich sein werden, die mechanistische Ansätze mit Methoden aus der künstlichen Intelligenz (KI) kombinieren.

Onkologe 2019 25 (Suppl 1):S109-S115 https://doi.org/10.1007/s00761-019-00652-1 (c) Der/die Autor(en) 2019

S. Schade · L. A. Ogilvie - T. Kessler · M. Schütte · C. Wierling • B. M. Lange · H. Lehrach · M.-L. Yaspo

\section{Ein daten- und modellgesteuerter Ansatz zur Behandlung maligner Tumoren}

Zusammenfassung

Alle Menschen sind einzigartig und so sind es auch ihre Krankheiten. Genome, Erkrankungsverläufe, Verhalten und Lebensstile sind unterschiedlich; es ist daher nicht erstaunlich, dass Menschen auch auf dieselben Medikamente unterschiedlich reagieren. Gerade Krebserkrankungen sind komplex und heterogen: Sie entstehen in Patienten mit individuellen Genomen und in Zellen mit je eigenen Epigenomen, sie entwickeln und entfalten sich auf der Basis zufälliger Prozesse. Dabei hängt das Ansprechen auf eine Therapie nicht nur von der einzelnen Tumorzelle, sondern auch von vielen patienteneigenen Faktoren ab. Die Entscheidung für eine optimale Behandlung erfordert daher eine tiefgehende molekulare Analyse, die sowohl den Patienten als auch den Tumor einschließt (z. B. „comprehensive molecular tumor analysis" [CMTA]) und anhand derer sich das Ansprechen auf mögliche personalisierte Therapien deutlich besser vorhersagen lässt.
Zurzeit befinden wir uns an einem Wendepunkt: Technologische Fortschritte, verringerte Kosten für Sequenzierung und andere molekulare Analysen sowie Weiterentwicklungen in der Datenverarbeitung können jetzt zusammengeführt werden und werden zum Fundament für den Aufbau eines datengesteuerten Ansatzes in der personalisierten Onkologie. Der Beitrag diskutiert die umfassende molekulare Charakterisierung einzelner Tumoren und Patienten als Basis nicht nur für die aktuelle Präzisionsonkologie, sondern auch für Rechenmodelle („digitale Zwillinge“), welche die Grundlage einer künftig tatsächlich personalisierten Therapieauswahl bilden werden.

Schlüsselwörter

Präzisionsmedizin · Tumorbiomarker . Genexpressionsanalyse · Translationale medizinische Forschung · Molekulare zielgerichtete Therapie

\section{A data- and model-driven approach for cancer treatment. German version}

\section{Abstract}

We are unique and so are our diseases. Our genomes, disease histories, behavior, and lifestyles are all different. It is not too surprising, therefore, that we often respond differently to the drugs we receive. Cancer, in particular, is a complex and heterogeneous disease, originating in patients with different genomes, in cells with different epigenomes, formed and evolving on the basis of random processes, with the response to therapy not only depending on the individual cancer cell but also on many features of the patient. Selection of an optimal therapy will therefore require a deep molecular analysis comprising both the patient and their tumor (e.g., comprehensive molecular tumor analysis [CMTA]), and much better personalized prediction of response to possible therapies.
Currently, we are at an inflection point in which advances in technology, decreases in the costs of sequencing and other molecular analyses, and increases in computing power are converging, forming the foundation to build a data-driven approach to personalized oncology. Here, we discuss the deep molecular characterization of individual tumors and patients as the basis of not only current precision oncology but also of computational models ('digital twins'), forming the foundation of a truly personalized therapy selection of the future.

\section{Keywords}

Precision medicine - Biomarkers, tumor - Gene expression profiling · Translational medical research - Molecular targeted therapy 


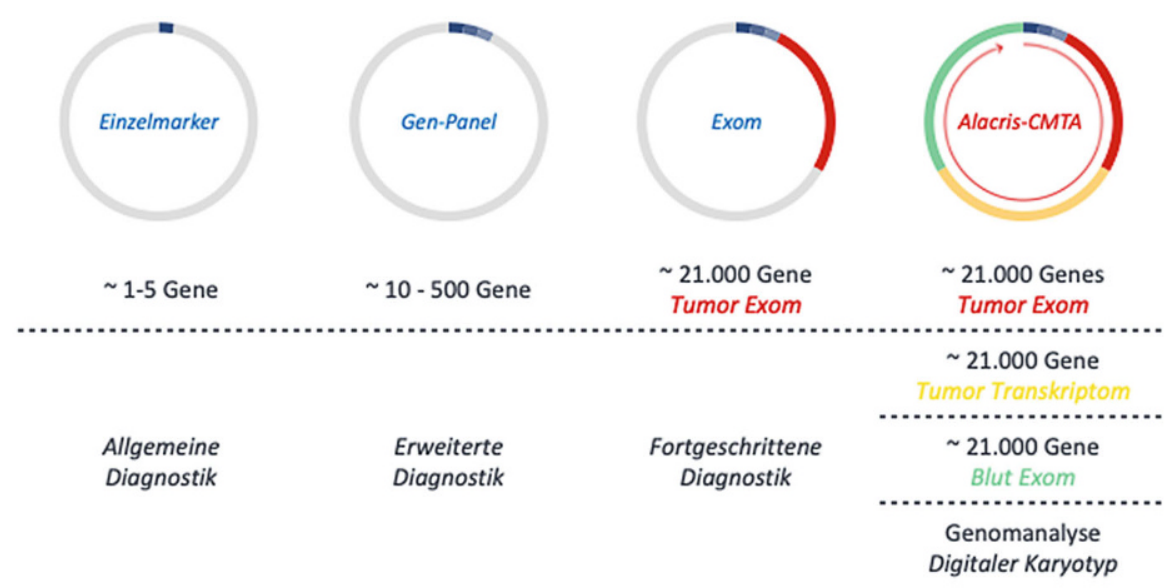

Abb. $1<$ Vergleich verschiedener diagnostischer Ansätze. (Adaptiert aus [11], mit freundl. Genehmigung S. Karger, Basel, Schweiz)
Gleichzeitig mit der Erweiterung der Wissensbasis zu Tumorerkrankungen, zellulärer Signalverarbeitung und molekularen Interaktionen erweitern sich auch unsere Möglichkeiten, Computermodelle $\mathrm{zu}$ erstellen, die in der Lage sind, die komplexen Netzwerke und die Cross-Talk-Prozesse, welche die Tumorprogression und das Ansprechen auf Medikamente beeinflussen, genau abzubilden [16-21]. Insbesondere mechanistische Modelle basierend auf Differenzialgleichungssystemen gehören zu den vielversprechendsten Ansätzen, um das dynamische Verhalten komplexer zellulärer Prozesse im KrebsKontext quantitativ zu erfassen und individualisierte Vorhersagen über die Wirksamkeit von Medikamenten zu ermöglichen [18-21]. Um die Wirkung eines Wirkstoffs auf einen bestimmten Tumor $\mathrm{zu}$ simulieren, muss ein mechanistisches Modell, welches das Wissen über relevante Tumorsignalwege und detaillierte mechanistische Wirkstoffdaten integriert, mithilfe der molekularen Informationen über Tumor und Patienten (funktional relevante Sequenzvarianten oder Genfusionen, Expressionsänderungen, etc.) personalisiert werden. Relevante Änderungen werden dann verwendet, um die funktionalen Merkmale oder die Konzentration der entsprechenden Objekte im Modell $[18,19] \mathrm{zu}$ modifizieren.
Die wesentlichen Vorteile mechanistischer Modelle liegen in der Integration von Daten aus verschiedenen Quellen und experimentellen Protokollen sowie in der Möglichkeit, Hypothesen für kausale Mechanismen durch das Design von In-silico-Experimenten zur Beantwortung noch offener Fragen zu generieren $[22,23]$. Die Beeinflussung einzelner Komponenten innerhalb des mechanistischen Netzwerks (z.B. durch Simulation von Mutationen oder Veränderungen in der Genexpression) ermöglicht die Untersuchung funktioneller Effekte auf verschiedene Signaltransduktionswege und die Identifikation von Komponenten, die aussichtsreiche Behandlungsziele darstellen.

Darüber hinaus können Medikamente virtuell gescreent werden unter Einbeziehen des patienteneigenen Backgrounds. So lässt sich zwischen wirksamen und nichtwirksamen Medikamenten differenzieren und die Auswahl der optimalen Dosierung sowie die Vorhersage von Off-target-Effekten, die $\mathrm{zu}$ schweren Nebenwirkungen führen können (• Abb. 2; [22, 24]), erreichen.

Damit stellt die mechanistische Modellierung einen leistungsfähigen, vielversprechenden Ansatz für virtuelle klinische Studien, die Identifikation von Wirkstoff-Targets und für die personalisierte Medizin dar (• Abb. 2; s. auch [18-20, 24]).

\section{Herausforderung Modell- parametrisierung}

Zwar ist die Struktur dieser Modelle auf der Basis jahrzehntelanger Grundlagenforschung gut definiert, doch über die kinetischen Konstanten und andere Parameter in der komplexen Umgebung von Zellen und Organismen ist wenig bekannt. Gerade diese Werte sind jedoch für quantitative Vorhersagen absolut unverzichtbar. Um diese Art von Parametern abzuschätzen, müssen wir die Parameter des Systems iterativ ableiten, indem wir die Unterschiede zwischen Modellvorhersagen und experimentellen Daten minimieren. Aus unserer Sicht stellt dies den größten verbleibenden Engpass auf dem Weg zu einer daten- und modellgesteuerten personalisierten Medizin der Zukunft dar. Aber die Bewältigung dieser Herausforderung wird einen großen Schritt nach vorn bedeuten, um die Reaktion eines Individuums auf zielgerichtete Krebsmedikamente genau vorherzusagen, sodass sich personalisierte Computermodelle direkt einsetzen lassen, um optimale Therapieentscheidungen treffen zu können.

\section{Von rein mechanistischen Modellen zu Hybridmodellen}

In vielen anderen Bereichen (z. B. Wettervorhersagen, virtuelle Crashtests) ha- 


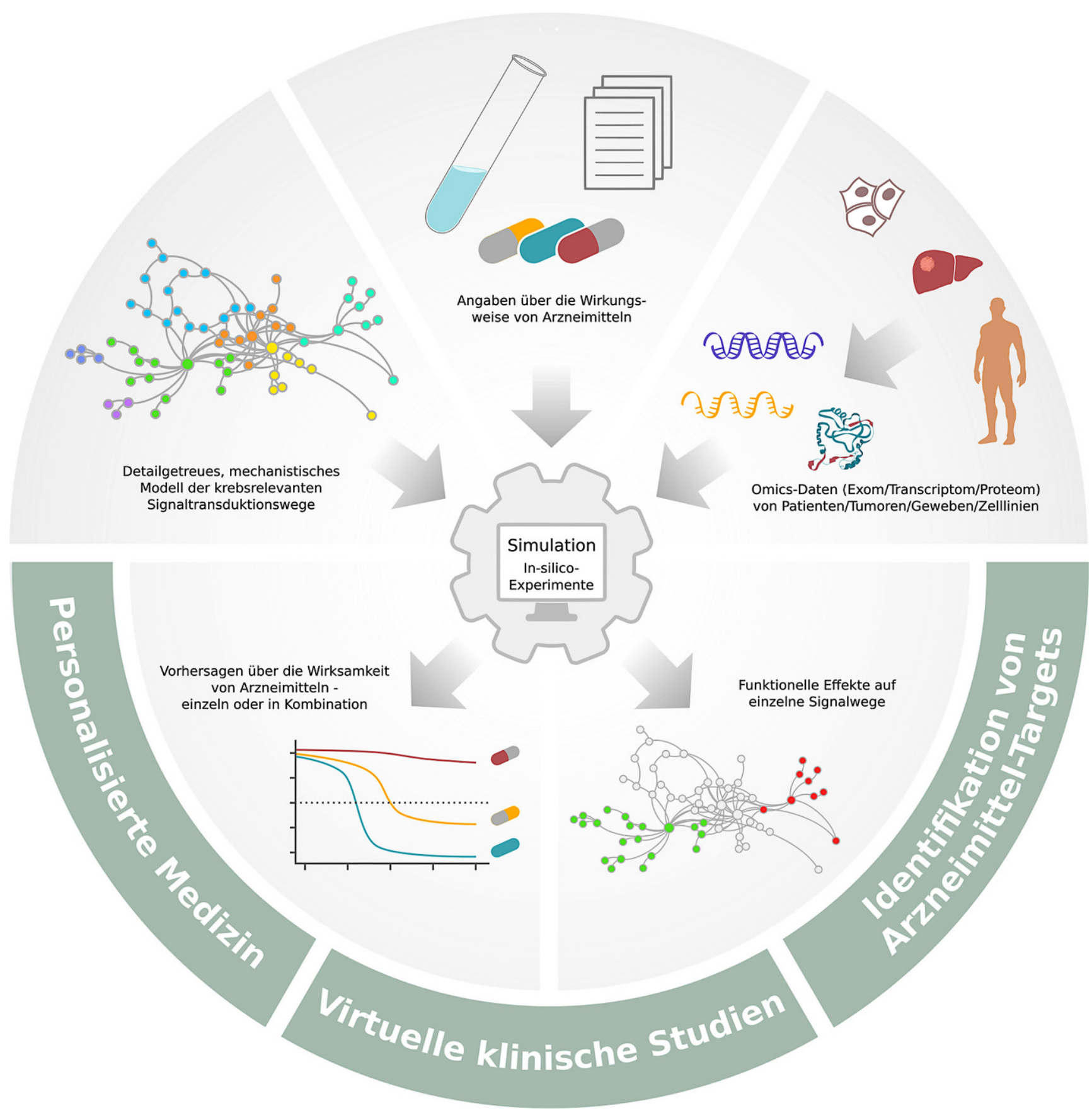

Abb. $2 \Delta$ Workflow und Anwendungen der mechanistischen Modellierung

ben sich mechanistische Computermodelle schon als hervorragende Werkzeuge erwiesen, die eine Vielzahl von Daten integrieren können. Für Prozesse, zu deren genauen molekularen Mechanismen keine Informationen vorliegen, könnten Hybridmodelle, die mechanistische Modellkomponenten mit klassischen KI-Techniken (z. B. neuronale Netze) kombinieren, von Nutzen sein, indem sie die Stär- ken beider Strategien vereinen (und dabei deren Schwachstellen vermeiden).

Von der Modellierung des Tumors zur Modellierung des Patienten: das DigiTwinsKonzept

Wirkungen wie Nebenwirkungen eines oral einzunehmenden Krebsmedikaments hängen von einer Reihe von Fak- toren $\mathrm{ab} . \mathrm{Zu}$ diesen zählen die enorme biologische Komplexität der Tumorzellen und die Heterogenität des Tumors, die Verstoffwechselung des Medikaments durch die intestinale Mikrobiota, die vom Genom des Patienten bestimmte Pharmakogenomik, mögliche Nebenwirkungen auf relevante Zellarten und, insbesondere für die Immuntherapie, das Immunsystem des Patienten. Daher wird es von zentraler Bedeutung sein, nicht 


\section{Infobox \\ - www.digitwins.org \\ - https://ipc-project.eu/mailto:info@alacris. de}

mehr nur den Tumor zu modellieren, sondern auch die relevanten Gewebeund Zelltypen des Patienten, die miteinander kommunizieren (in diesem Fall die Konzentration der aktiven Arzneimittelformen im zeitlichen Verlauf), und die komplexen Wechselwirkungen des Tumors mit dem Immunsystem. Dieses Konzept kann auf jeden anderen Bereich von Erkrankungen übertragen werden. Durch die Modellierung relevanter Komponenten und Prozesse im einzelnen Patienten sowie deren Interaktionen werden virtuelle Patienten konstruiert. So lassen sich eine tatsächlich personalisierte Therapieauswahl und Prävention sowie eine Palette von gesundheitsfördernden Maßnahmen entwickeln, nicht nur für eine wachsende Anzahl von Patienten, sondern auch für gesunde Menschen (s. http://www.digitwins.org).

\section{Ausblick}

Medikamente beeinflussen die komplexen und hochvariablen biologischen Netzwerke in unserem Körper. Um das Ansprechen eines konkreten Patienten bzw. Tumors auf ein Medikament vorherzusagen, müssen deshalb die relevanten biologischen Netzwerke äußerst detailliert charakterisiert werden. Noch vor kurzem war ein solcher Ansatz undenkbar oder schlicht zu teuer, er war finanziell deutlich aufwendiger als der klassische pathologiebasierte Ansatz für Diagnose und empirische Therapieauswahl. Durch Weiterentwicklungen in einer Reihe von Bereichen, von der Sequenzierung der nächsten Generation (NGS) sowie anderen Omics-, bildgebenden und sensorgestützten Methoden bis hin zur Informatik, lässt sich dieser Ansatz jedoch immer mehr umsetzen.

Die Möglichkeit, die Auswirkungen eines Medikaments oder einer Medikamentenkombination auf den einzelnen Patienten in silico vorherzusagen, eröffnet zahlreiche neue Möglichkeiten für die Zukunft der Präzisionsonkologie. Mit der Erhöhung der Wahrscheinlichkeit, dass der einzelne Patient eine wirksame Behandlung erhält, werden die positiven Auswirkungen nicht nur für den Patienten, sondern auch für die Gesundheitssysteme überhaupt spürbar sein. Durch die Verbesserung von Behandlungsergebnissen und von Lebensqualität wird eine potenzielle Verringerung der Therapiekosten sowie der rezidiv- und endstadiumbedingten klinischen Kosten erwartet. Diese wiederum würde einen potenziellen Anstieg der diagnostischen (Omics-basierte Medizin) Kosten ausgleichen (die NGS-Kosten verringern sich bereits jetzt rasant) und durch eine effektivere Steuerung des Ressourceneinsatzes den Abbau von Ungleichheiten in Gesundheitssystemen fördern.

Der von uns beschriebene daten- und modellgesteuerte Ansatz zur Behandlung von Krebspatienten ist nicht Teil unserer fernen Zukunft, sondern liegt in Reichweite: klinische Proof-of-principle-Pilotstudien, wie Treat20plus, und intensive Forschungsarbeit sind bereits jetzt in vollem Gange (z.B. iPC, ,individualized pediatric cure“, ein Projekt des Forschungs- und Innovationsprogramms Horizon 2020 der Europäischen Union, https://ipc-project.eu). Insbesondere das Projekt Treat20plus hebt die nahtlose Integration dieses Ansatzes in den bestehenden Versorgungsrahmen hervor. Seine Ergebnisse tragen zu den Diskussionen molekularer Tumorboards und zur anschließenden Behandlungsentscheidung bei. Im weiteren Kontext suchen Initiativen wie DigiTwins (www. digitwins.org) nach einer nachhaltigen, auf einem daten- und modellgesteuerten Ansatz basierenden Zukunftsvision für die Gesundheitsversorgung. Der Schlüssel zu dieser Vision liegt in den technologischen Entwicklungen. Die routinemäßige Integration von Technologien wie Single-cell-NGS [25] und Insitu-Sequenzierung [26] sowie sensorbasierter und bildgebender Verfahren wird entscheidend dafür sein, dass Patienten und deren Tumore detailliert genug charakterisiert werden können, und sie wird künftigen Modellen als grundlegender Daten-Input dienen.

\section{Klinische Relevanz}

- Tumorboards in den Kliniken können durch einen daten- und modellgetriebenen Ansatz im Rahmen des aktuellen Patientendiagnose- und Therapieauswahlverfahrens informiert werden.

- Schon jetzt wird der daten- und modellgesteuerte Ansatz der personalisierten Onkologie in klinischen Pilotstudien getestet.

- Um Spezifität, Genauigkeit und Sensitivität von Vorhersagen mit Modellierungen sicherzustellen, sind weitere Optimierung und Entwicklungen erforderlich.

\section{Korrespondenzadresse

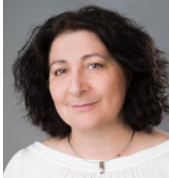 \\ Dr. Marie-Laure Yaspo \\ Alacris Theranostics $\mathrm{GmbH}$ \\ Max-Planck-Str. 3 \\ 12489 Berlin, Deutschland m.yaspo@alacris.de}

Danksagung. Die Autoren danken Romy Kümpfel und dem weiteren Alacris Theranostics Team für die Hilfe bei der Erstellung des Manuskripts. Diese Arbeit wurde vom Bundesministerium für Bildung und Forschung (BMBF) unter der Förderkennzahl 031A512C (Treat20plus) gefördert.

Funding. Open access funding provided by Max Planck Society.

\section{Einhaltung ethischer Richtlinien}

Interessenkonflikt. S. Schade, L.A. Ogilvie, T. Kessler, M.Schütte, C. Wierling, B.M. Lange und M.-L. Yaspo sind Mitarbeiter der Alacris Theranostics GmbH. H. Lehrach ist Vorstandsmitglied der Alacris Theranostics $\mathrm{GmbH}$.

Für diesen Beitrag wurden von den Autoren keine Studien an Menschen oder Tieren durchgeführt.

Open Access. Dieser Artikel wird unter der Creative Commons Namensnennung 4.0 International Lizenz (http://creativecommons.org/licenses/by/4.0/deed. de) veröffentlicht, welche die Nutzung, Vervielfältigung, Bearbeitung, Verbreitung und Wiedergabe in jeglichem Medium und Format erlaubt, sofern Sie den/die ursprünglichen Autor(en) und die Quelle ordnungsgemäß nennen, einen Linkzur Creative Commons Lizenz beifügen und angeben, ob Änderungen vorgenommen wurden. 


\section{Literatur}

1. World Health Organisation, Data and Statistics: http://www.euro.who.int/en/health-topics/ noncommunicable-diseases/cancer/data-andstatistics

2. Luengo-Fernandez R, Leal J, Gray A et al (2013) Economic burden of cancer across the European Union: a population-based cost analysis. Lancet Oncol 1(2):1165-1174

3. Slamon D, Eiermann W, Robert $\mathrm{N}$ et al (2011) Adjuvant trastuzumab in HER2-positive breast cancer. N Engl J Med 365(14):1273-1283

4. Paplomata E, Nahta R, O'Regan RM (2015) Systemic therapy for early-stage HER2-positive breast cancers: Time for a less-is-more approach? Cancer 121(4):517-526

5. de Gramont A, Watson S, Ellis LM (2014) Pragmatic issues in biomarker evaluation for targeted therapies in cancer. Nat Rev Clin Oncol 12(4):197-212

6. Dagogo-Jack I, Shaw AT (2018) Tumour heterogeneity and resistance to cancer therapies. Nat Rev Clin Oncol 15:81-94

7. Hovestadt V, Jones DTW, Picelli S et al (2014) Decoding the regulatory landscape of medulloblastoma using DNA methylation sequencing. Nature 510(7506):537-541

8. Weischenfeldt J, Simon R, Feuerbach L et al (2013) Integrative genomic analyses reveal an androgendriven somatic alteration landscape in early-onset prostate cancer. Cancer Cell 23(2):159-170

9. van't Veer L, Yau C, Yu NY et al (2017) Tamoxifen therapy benefit for patients with 70-gene signature high and low risk. Breast Cancer Res Treat 166(2):593-601

10. Sultan M, Schulz MH, Richard Het al (2008) A global view of gene activity and alternative splicing by deep sequencing of the human transcriptome. Science 321:956-960

11. Schütte M, Ogilvie LA, Rieke DT et al (2017) Cancer precision medicine: Why more is more and DNA is notenough. Public Health Genomics 20(2):70-80

12. Snyder A, Makarov V, Merghoub T et al (2014) Genetic basis for clinical response to CTLA-4 blockade in melanoma. NEngl J Med 371(23):2189-2199

13. Topalian SL, Taube JM, Anders RA, Pardoll DM (2016) Mechanism-driven biomarkers to guide immune checkpoint blockade in cancer therapy. Nat Rev Cancer 16(5):275-287

14. SicklickJK, Kato S, Okamura Retal (2019) Molecular profiling of cancer patients enables personalized combination therapy: The I-PREDICT study. Nat Med 25(5):744-750

15. Devulapally PR, Bürger J, Mielke T et al (2018) Simple paired heavy-and light-chain antibody repertoire sequencing using endoplasmic reticulum microsomes. Genome Med 10(1):34

16. Kolch W, Halasz M, Granovskaya M et al (2015) The dynamic control of signal transduction networks in cancer cells. Nat Rev Cancer 15(9):515-527

17. Tyson JJ, Baumann WT, Chen Cetal (2011) Dynamic modelling of oestrogen signalling and cell fate in breast cancer cells. Nat Rev Cancer 11(7):523-532

18. Wierling C, Kessler T, Ogilvie LAetal (2015) Network and systems biology: essential steps in virtualising drug discovery and development. Drug Discov Today Technol 15:33-40

19. Wierling C, Kühn A, Hache H et al (2012) Prediction in the face of uncertainty: A Monte Carlo-based approach for systems biology of cancer treatment. MutatRes 746(2):163-170

20. Fröhlich F, Kessler T, Weindl D et al (2018) Efficient Parameter Estimation Enables the Prediction of
Drug Response Using a Mechanistic Pan-Cancer Pathway Model. Cell Syst.7(6), 567-579.e566

21. Röhr C, Kerick M, Fischer A et al (2013) Highthroughput miRNA and mRNA sequencing of paired colorectal normal, tumor and metastasis tissues and bioinformatic modeling of miRNA-1 therapeutic applications. PLoS ONE 8(7):e67461

22. Clegg L, Mac Gabhann F (2015) Molecular mechanism matters: Benefits of mechanistic computational models for drug development. Pharmacol Res 99:149-154

23. Baker R, Peña J, Jayamohan J et al (2018) Mechanistic models versus machine learning, a fight worth fighting for the biological community? Biol Lett 14(5):20170660

24. Lehrach $H$ (2015) Virtual clinical trials, an essential step in increasing the effectiveness of the drug development process. Public Health Genomics 18(6):366-371

25. Haque A, Engel J, Teichmann SA et al (2017) A practical guide to single-cell RNA sequencing for biomedical research and clinical applications. Genome Med 9:75

26. Lee JH, Daugharthy ER, Scheiman J et al (2014) Highly multiplexed subcellular RNA sequencing in situ. Science 343(6177):1360-1363 